\title{
Spontaneous pregnancy in a patient with premature ovarian insufficiency - case report
}

\author{
Anna Calik-Ksepka, Monika Grymowicz, Weronika Bronkiewicz, Aleksandra Urban, Kamil Mierzejewski, \\ Ewa Rudnicka, Roman Smolarczyk
}

Department of Gynaecological Endocrinology, Medical University of Warsaw, Warsaw, Poland

\begin{abstract}
Diagnosis of premature ovarian insufficiency is usually sudden and distressful for the patient in terms of facing infertility. However, some patients with POI have intermittent ovarian activity with an overall $5 \%$ probability of pregnancy. We report the case of 27 -year-old woman with premature ovarian insufficiency treated with hormone replacement therapy, who six months after diagnosis conceived spontaneously. Apart from bleeding episodes in the first trimester, the pregnancy course was uneventful, and a healthy neonate was delivered. Women with premature ovarian insufficiency should be informed about the small but nonetheless real possibility of pregnancy.
\end{abstract}

Key words: premature ovarian insufficiency, spontaneous pregnancy.

\section{Introduction}

Premature ovarian insufficiency (POI) affects approximately $1 \%$ of women under the age of 40 years and is associated with female infertility [1]. The symptoms are similar to those of menopause: oligo/amenorrhea, hypoestrogenism, elevated level of gonadotropins, and diminished number of follicles within the ovaries. Although the causes of POI are largely unknown, genetic defects such as Turner syndrome and fragile $X$ syndrome premutation, environmental factors, and autoimmune diseases seem to play an important role in the aetiopathology.

Primary ovarian insufficiency is generally diagnosed as part of the evaluation for frequent gynaecological complaints, such as irregular menses, so the diagnosis usually comes unexpectedly. Besides causing infertility, POI is associated with multiple health risks such as menopausal symptoms, decreased bone mineral density and increased risk of fractures, greater risk of cardiovascular diseases, and psychological impact, which may include depression and potential early decline in cognition. Ovarian failure is not permanent, which differentiates this condition form menopause. Women with POI ovulate extremely rarely. However, $5 \%$ of such women conceive spontaneously and have a normal pregnancy after the diagnosis is established [2].

\section{Case report}

A 27-year-old woman was referred to the gynaecological endocrinology clinic with oligomenorrhoea, and periodic stomach and spine pain. Menarche occurred at age of 16 years, followed by regular menstrual bleeding. At 19 years, she commenced on birth control pills that she continued for three years. Her personal history for previous surgery or other relevant pathologies was negative. Laboratory tests showed elevated folliclestimulating hormone (FSH) level at $102.2 \mathrm{mIU} / \mathrm{ml}$ (normal levels 3.03-8.08 $\mathrm{mlU} / \mathrm{ml}$ ), oestradiol (E2) at $<10 \mathrm{pg} / \mathrm{ml}(21-251 \mathrm{pg} / \mathrm{ml})$, and luteinising hormone (LH) $45.9 \mathrm{mIU} / \mathrm{ml}$ (1.8-11.78 mlU/ml). Repeated laboratory results confirmed the diagnosis of premature ovarian insufficiency. Anti-Müllerian hormone (AMH) was at the very low level of $0.01 \mathrm{ng} / \mathrm{ml}$. Thyroid-stimulating hormone (TSH), prolactin, and androgen status were within normal limits. Lower pelvic transvaginal ultrasonography found no pathology. The patient's karyotype corresponded to the normal female karyotype.

Hormone replacement therapy (HRT) was implemented to minimise bone loss and decrease the risk of cardiovascular events. The patient was treated with cyclical hormone therapy using oestrogen $(2 \mathrm{mg}$ oestradiol) with the addition with progestin for 14 days in the cycle (10 mg dydrogesterone). Our patient sponta- 
neously and unexpectedly conceived after six months on HRT. After recognising the pregnancy, HRT treatment was discontinued. During the first trimester of pregnancy several episodes of vaginal bleeding occurred. Progesterone therapy was then implemented. Pregnancy proceeded without any further complications. At the $39^{\text {th }}$ week of gestation a healthy child was born.

\section{Conclusions}

Diagnosis of POI is usually sudden and very distressing for the patient. Due to a diminished number of follicles, the patients face the prospect of infertility. Oocyte donation is the only proven and recommended treatment for infertility in women with POI. Cumulative pregnancy rates of oocyte donation treatment are very high (70-80\%). However, such treatment is not available in some countries, and not all patients would accept oocyte donation.

However, while normal menopause is generally an irreversible event, $\mathrm{POI}$ is characterised by intermittent ovarian function with oestrogen production and even ovulation, despite the presence of high gonadotropin levels. Spontaneous ovulations were observed in $20-24 \%$, and conceptions in about $5 \%$, of women with POI $[3,4]$. It is important to admit the probability of ovulation occurrence in the case of patients with oligo/amenorrhoea. The chances of resuming ovarian function depend chiefly on whether the amenorrhoea is primary or secondary. Large studies have shown that numerous mechanisms responsible for the primary amenorrhoea are more advanced and serious than in the case of secondary amenorrhoea $[4,5]$. Unexpectedly, the aetiologies responsible for familial forms of POI may show an increased probability of resuming ovarian function [4].

Another important predictor of resumption of ovarian activity are the results of laboratory tests of FSH, oestradiol, and inhibin B levels [4, 5]. AMH levels prove not to be predictive of resumption of ovarian activity in $\mathrm{POI}$ women [5]. It is crucial to underline the importance of educating and reassuring patients that spontaneous pregnancies in women with $\mathrm{POI}$ are not associated with higher obstetric morbidity or neonatal risk as compared with the general population, and may, just like in the presented case, lead to the birth of a healthy child. It is also important to inform patients who do not want to get pregnant about the necessity of contraception usage, because of the risk of pregnancy as a result of spontaneous resumption of ovarian activity.

\section{Disclosure}

The authors report no conflict of interest.

\section{References}

1. Rebar RW. Premature ovarian failure. Obstet Gynecol 2009; 113: 13551363.

2. Nelson LM. Clinical practice. Primary ovarian insufficiency. N Engl J Med 2009; 360: 606-614.

3. Komorowska B. Autoimmune premature ovarian failure. Menopause Rev 2016; 15: 210-214.

4. Bidet $M$, Bachelot $A$, Bissauge $E$, et al. Resumption of ovarian function and pregnancies in 358 patients with premature ovarian failure. J Clin Endocrinol Metab 2011; 96: 3864-3872.

5. Tucker EJ, Grover SR, Bachelot A, et al. Premature ovarian insufficiency: new perspectives on genetic cause and phenotypic spectrum. Endocr Rev 2016; 37: 609-635. 